\title{
ANÁLISIS
}

\section{Las políticas de integración económica en el Pacífico asiático y el Pacífico latinoamericano: una perspectiva mexicana}

DOI: $10.32870 /$ mycp.v2i5.409

Roberto Hernández Hernández ${ }^{1}$

\section{Resumen}

En este trabajo se analizan las políticas de integración económica puestas en práctica por los actores internacionales involucrados en la negociación de tres instrumentos en construcción: el Tratado Transpacífico de Asociación Económica Estratégica (TPP), el Regional Comprehensive Economic Partnership (RCEP), ambos en el Pacífico asiático, y la Alianza del Pacífico (AP) en el Pacífico americano.

En el documento se plantea la perspectiva de México respecto de su participación activa, tanto en el TPP como en la Alianza del Pacífico, así como la necesidad de dar seguimiento, de manera permanente y sistemática, a otros procesos de regionalización de importancia global como el RCEP y el TLC entre Estados Unidos y la Unión Europea.

Artículo recibido el 04 de julio de 2013 y dictaminado el 01 de agosto de 2013.

1. Profesor-investigador del Departamento de Estudios del Pacífico y del Departamento de Estudios Internacionales, del Centro Universitario de Ciencias Sociales y Humanidades de la Universidad de Guadalajara. ORCID http://orcid.org/0000-0002-9145-5865 
La investigación se fundamenta, principalmente, en las herramientas teóricas de la economía política internacional y el constructivismo tradicional. En este sentido, los temas tratados se abordan desde una perspectiva del análisis de multinivel (poder económico, poder militar y actores internacionales no gubernamentales), pero poniendo énfasis en el nivel económico; los aspectos militares y el papel de los actores internacionales transfronterizos se abordan de manera complementaria. ${ }^{2}$

Palabras clave: México, China, Estados Unidos, Pacífico asiático, TPP, RCEP.

\section{Abstract}

This paper analyzes the economic integration policies implemented by economies of both shores of the Pacific basin through three instruments under negotiation: the Trans-Pacific Partnership Agreement (TPP), the Regional Comprehensive Economic Partnership (RCEP), and Pacific Alliance (AP).

The paper investigates Mexico's participation in the TPP and the AP. It also points out the importance for the Mexican economy's relationship between AP and TPP with the RCEP. Referentially analyze the correlation between these three instruments with economic regionalization projects in the Americas and Europe, including new free trade agreements.

The research is based mainly on the theoretical tools of international political economy and traditional constructivism. In this sense, the issues are addressed from a multilevel analysis (economic, military and international non-governmental actors), but with an emphasis on the economic level. The military subjects and role of transnational actors are addressing on complementary manner.

Keywords: Mexico, China, United States, TPP, RCEP, AP.

\section{Introducción}

Somos testigos del gran impulso dado por los gobiernos de todo el mundo a la negociación de tratados preferenciales de comercio e inversión (conocidos como tratados de libre comercio) de carácter bilateral y regional. Estos ins-

2. El autor agradece el apoyo de Fabián Tostado Ávila en la recopilación de información sobre el caso de la Alianza del Pacífico. 
trumentos de cooperación económica se han convertido en premisa básica para la integración regional.

Después del significativo avance en los procesos de integración que durante más de medio siglo han realizado los países europeos, en los últimos lustros el Este de Asia y América Latina se han empeñado en encontrar nuevas modalidades de integración institucional que les permitan cumplir con los objetivos propuestos por la Organización Mundial de Comercio (OMC) desde su fundación. En este sentido, los dirigentes (sobre todo gubernamentales y empresariales) de los países que conforman estas áreas geográficas están tomando las decisiones político-económicas que podrían propiciar la configuración de un nuevo paradigma de integración económica interregional, con grandes implicaciones para el sistema internacional y sus actores fundamentales: el Estado, las empresas transnacionales y la sociedad civil. ${ }^{3}$

Al analizar la política exterior de los jugadores estratégicos en el sistema internacional y relacionarla con los temas que se negocian en el contexto de los esquemas de regionalización, podemos inferir que 2013 y 2014 serán cruciales para la reconfiguración de los procesos de integración económica regional, dada su influencia en los campos del comercio, inversión y organización productiva de muchos países de Asia, América y Oceanía.

En este trabajo se analizan las políticas de integración económica de tres megatratados regionales en proceso de negociación: el Tratado Transpacífico de Asociación Económica Estratégica (TPP), la Asociación Económica Integral Regional (Regional Comprehensive Economic Partnership) (RCEP) y la Alianza del Pacífico (AP). A nivel de contextualización y como referencia obligada se toman en cuenta otros mecanismos y proyectos de cooperación económica regional, tales como la Unión Europea, el Tratado de Libre Comercio de América del Norte (TLCAN), el Mercado Común del Sur (Mercosur), la Alianza Bolivariana para América (ALBA), la Asociación de Libre Comercio de las Américas (ALCA) y el Tratado de Libre Comercio de APEC (FTAAP).

3. De conformidad con los planteamientos de Jürgen Habermas, por sociedad civil se entiende al conjunto de instituciones que definen y defienden los derechos individuales, políticos y sociales de los ciudadanos, las cuales propician su libre asociación, la posibilidad de defenderse de la acción estratégica del poder y del mercado, y la viabilidad de intervenir en la operación misma del sistema. En este sentido se incluye entre los componentes de la sociedad civil a las organizaciones no lucrativas, organizaciones no gubernamentales, asociaciones y fundaciones, incluyendo a las universidades, colegios profesionales y comunidades religiosas. 
En el documento se plantea la posición oficial de México respecto de su participación activa tanto en el TPP como en la Alianza del Pacífico; así como la importancia que representan para el país los procesos de cooperación regional en Asia-Pacífico (en su vertiente asiática y panamericana). Además, la participación en otros foros de integración latinoamericana y el seguimiento puntual a los procesos de integración como RCEP y las negociaciones del TLC entre Estados Unidos y la Unión Europea, constituyen variables estratégicas para reorientar las políticas económicas de México, dada la estrecha relación existente entre los procesos de regionalización en marcha y el compromiso manifiesto de México "con el libre comercio, la movilidad de capitales y la integración productiva” (PND, 2013: 92).

Esta investigación se fundamenta principalmente en las herramientas teóricas de la economía política internacional y el constructivismo tradicional. En este contexto, los temas tratados se abordan desde una perspectiva del análisis de multinivel como el planteado por Nye (2004 y 2013), ${ }^{4}$ poniendo énfasis en el nivel intermedio, relacionado con el poder económico. Los aspectos del poder militar y el papel de los actores internacionales transfronterizos se refieren de manera complementaria.

\section{El Pacífico asiático}

Las políticas de integración económica aplicadas actualmente en el Pacífico asiático, que tienen como centro al Este de Asia, están contribuyendo de manera significativa a redefinir las relaciones comerciales y productivas de los actores regionales entre sí, y en un sentido más amplio a rediseñar sus relaciones económicas con el resto del mundo. Dos megatratados regionales son los que actualmente se encuentran en plena negociación: el Tratado Transpacífico de Asociación Económica Estratégica (TPP), liderado por Estados Unidos, y la Asociación Económica Integral Regional (Asian Regional Comprehensive Economic Partnership - RCEP-), que toma como centro de

4. Joseph Nye plantea que en el mundo actual, la distribución del poder varía según el contexto. Se distribuye en un patrón que se asemeja a un juego de ajedrez tridimensional. En el tablero superior se encuentra el poder militar, el cual en gran medida es unipolar y es sustentado por Estados Unidos. En el nivel medio se encuentra el poder económico, el cual desde hace más de una década es multipolar, siendo Estados Unidos, Europa, China y Japón los principales jugadores. En la parte inferior del tablero están los actores internacionales transfronterizos que actúan fuera del control de los gobiernos (Nye, 2004: 4; Nye, 2013). 
negociación los tratados regionales ASEAN+1, y es liderado por China. ${ }^{5}$ Dado que Estados Unidos, China y Japón son los socios más importantes de México, la participación mexicana en las negociaciones del TPP, y un seguimiento puntual de los avances del RCEP son de importancia estratégica, puesto que sus resultados impactarán su estructura productiva y sus relaciones comerciales con el exterior.

\section{Del Tratado Trans-Pacífico de Libre Comercio (P-4) al Tratado Transpacífico de Asociación Económica Estratégica (TPP). ¿El preámbulo al Tratado de Libre Comercio de APEC (FTAAP)?}

Las dificultades de los líderes del Este de Asia para encontrar una fórmula sobre su integración económica, dio tiempo para que Estados Unidos asumiera el liderazgo del proceso integrativo. Para ello propuso un mecanismo específico con objetivos y metas concretas, así como un proyecto de integración macrorregional a mediano plazo. Para ello tomaron como punto de partida la experiencia reciente del Pacific Four (P4), ${ }^{6}$ propusieron negociar de manera inmediata el Transpacific Partnership (TPP) y estudiar las posibilidades de configurar el Tratado de Libre Comercio de APEC (FTAAP) que incluiría a las 21 economías de APEC.

Estados Unidos se incorporó a las negociaciones del P4 sobre inversión y servicios financieros en marzo de 2008. Su participación activa en este instrumento cambió drásticamente la dimensión y los alcances del mismo. Meses después Australia, Perú, Vietnam y Malasia se integraron a las negociaciones.

5. Otro megatratado que se suma a los otros instrumentos bajo consideración en el Pacífico, es el US-EU FTA (the Transatlantic Trade and Investment Partnership (TTIP), lanzado oficialmente en la Cumbre del G8 en Irlanda el pasado 17 de junio.

6. El esquema del P4 fue lanzado en 2002 por el presidente de Chile y los primeros ministros de Nueva Zelanda y Singapur en Los Cabos, México, durante la Cumbre de Líderes de APEC. Meses después se integró Brunei. El tratado obtuvo su ratificación por parte de cada uno de los socios a lo largo de 2006 (Brewer, 2010). Los elementos fundamentales del P4 incluyen: el comercio de mercancías (más de $90 \%$ del comercio total que se realiza entre los cuatro países quedó libre de aranceles al momento de su ratificación), la facilitación de los intercambios de mercancías, la contratación pública, la cooperación en materia de competencia y los derechos de propiedad intelectual, así como la provisión de un marco para la colaboración en áreas estratégicas, como la innovación, investigación y desarrollo. Las negociaciones incluyeron, además de los aspectos comerciales, los temas de cooperación ambiental y un memorando de entendimiento sobre cooperación laboral, de carácter vinculante. Asimismo propusieron compartir sus conocimientos, ideas, tecnología y recursos para mejorar su competitividad en el mercado mundial (Ministerio de Relaciones Exteriores de Chile, 2006). 
Ya con el liderazgo de Estados Unidos, las partes convinieron en convertir al Tratado Trans-Pacífico de Libre Comercio (P-4) en el Tratado Transpacífico de Asociación Económica Estratégica (TPP).

Posteriormente, en noviembre de 2011, México, junto con Canadá, expresó durante la reunión de APEC en Honolulú, su interés por iniciar consultas con el resto de los socios para formar parte del proceso de negociación del TPP, proceso que se completó en octubre de 2012. Meses después lo haría Japón, para sumar los actuales 12 miembros. ${ }^{7}$

Una de las razones fundamentales de México para incorporarse a este mecanismo de integración económica regional fue el papel de liderazgo que adquirió Estados Unidos en cuanto a la conducción de las negociaciones. Dado el alto grado de vinculación entre la economía mexicana y la estadounidense, el costo de mantenerse al margen

[...] sería sumamente alto para México [...] Ello implicaría que México enfrente una mayor competencia de los miembros del TPP en el mercado de Estados Unidos y Canadá. Las exportaciones de Estados Unidos a otros mercados del TPP que usen insumos mexicanos tendrían que pagar arancel porque se perdería la posibilidad de acumulación de origen. En suma, México perdería competitividad y atractivo por captar flujos de inversión extranjera (Rosenzweig, 2012: 91 y 92). ${ }^{8}$

Durante las negociaciones han quedado claros los objetivos económicos del gobierno de Obama:

A través del TPP, Estados Unidos está tratando de avanzar en un tratado comercial y de inversión, de nueva generación, que mejorase la competitividad de Estados Unidos, la expansión del comercio estadounidense en la región Asia-Pacífico, y el estímulo a la creación y retención de empleos en Estados Unidos, mientras

7. Sobre un análisis comparativo de carácter económico y comercial de los miembros del TPP en relación con otros países de APEC, así como los tratados de libre comercio firmados por aquéllos, véase: Williams, R. Brock (2013), Trans-Pacific Partnership (TPP) Countries: Comparative Trade and Economic Analysis, Congressional Research Service, Washington.

8. Para explicar la incorporación de México al TPP, Rosenzweig menciona, además de la membresía de Estados Unidos en el acuerdo comercial, otras seis razones: la situación global poscrisis, dinamismo de las economías asiáticas, estado de las negociaciones a nivel multilateral, importancia económica que representa la región TPP, erosión de preferencias arancelarias para México y disciplinas cubiertas a nivel de ambición. Véase: Rosenzweig, Mendialdua Francisco L. (2012), "México y su ingreso al Acuerdo de Asociación Transpacífico", Revista de Derecho Económico Internacional, vol. 3, núm. 1, diciembre de 2012, pp. 89-96. 
que al mismo tiempo promueve los derechos laborales, la protección del medio ambiente y la transparencia (USTR, 2013).

En otras palabras, la propuesta de Estados Unidos y de otros países es lograr un tratado "integral y de alto estándar". Con la negociación y puesta en práctica del TPP se pretende liberalizar el comercio de casi todos los bienes y servicios y lograr compromisos más allá de los que demanda la OMC. De este modo, si el TPP se concluyera según el esquema previsto, podría eliminar las barreras arancelarias y no arancelarias al comercio y la inversión entre las partes y podría servir de modelo para un futuro acuerdo comercial entre los miembros de APEC y de otras regiones (Fergusson, 2013). Así, el TPP se presenta como un proceso de negociación con objetivos muy ambiciosos, dada la cobertura geográfica de los países participantes (de tres continentes) y una gran disparidad en sus respectivos niveles de desarrollo. Si a esta característica se le agrega la meta de concluir las negociaciones para octubre de 2013, en la Cumbre APEC de Bali, Indonesia, las dificultades de lograrlo son enormes. Incluso, de concluir exitosamente las negociaciones, faltaría el proceso de aprobación legislativa en cada uno de los respectivos países, el cual podría ser bastante lento, incluyendo el caso de Estados Unidos.

Sin menospreciar la importancia de los países que participan en las negociaciones, la capacidad de Estados Unidos se ve reflejada en el tipo de acuerdo que está perfilándose. Esto es, los intereses estadounidenses se ven mayormente representados en las discusiones. En este sentido, no es casual el énfasis que se ha puesto en promover cambios regulatorios en terceros países, tales como la mayor protección a las inversiones, menor control de capitales o los derechos laborales. El caso de la propiedad intelectual (PI) es quizá uno de los de mayor complejidad en las negociaciones, sobre todo por el interés manifiesto de incrementar los niveles de protección a los derechos de autor y a las patentes industriales (especialmente de la industria farmacéutica). Otros temas sensibles son las políticas de salud de cada uno de los países o la regulación de contenidos en Internet, que llevaría a revisar aspectos ya negociados en los tratados bilaterales (Cuevas, 2013).

Otras cuestiones clave que conviene mencionar, incluyen el acceso a mercados de productos muy sensibles, entre los que se cuentan los productos lácteos, azúcar, textiles y prendas de vestir, adquisiciones gubernamentales, inversiones, reglas de origen, competencia, mano de obra y normas ambientales, entre otras. En total, 29 capítulos están en discusión. Para los países 
menos desarrollados del grupo, las implicaciones económicas y políticas que tendrían que poner en práctica al aprobar los acuerdos alcanzados, son enormes (Fergusson, 2013).

Teniendo en cuenta las circunstancias señaladas, es entendible que después de la reunión de Lima, cuando se habían reportado "avances sustanciales" (USTR, 2013), las reuniones de Malasia y Brunei se hayan caracterizado por el estancamiento. No obstante, los 12 países que integran el grupo mantienen el objetivo de llegar a un acuerdo básico en la reunión de octubre próximo y concluir el tratado antes de que finalice 2013. Octubre es una fecha clave, dada la coincidente reunión de APEC, donde el mensaje sobre la viabilidad o inviabilidad del TPP tendría implicaciones sustanciales para la prevista ampliación del proyecto hacia la macrorregión de Asia-Pacífico.

Hay que tener en cuenta que el proyecto de extender el TPP para convertirlo en un Tratado de Libre Comercio de APEC se comenzó a perfilar en la cumbre anual de APEC de 2010. Allí los líderes de las 21 economías acordaron realizar los estudios de viabilidad necesarios para establecer el Área de Libre Comercio de APEC. Una señal positiva sobre este punto fue que ningún gobierno de la región impugnó la propuesta, aunque se reconocen los grandes obstáculos políticos y técnicos que habrá que superar para llevar a cabo su implementación. El FTAAP podría crear el tratado comercial más amplio de la historia. Los países miembros del APEC controlan la mitad del comercio mundial y representan $60 \%$ de la economía mundial. Ellos son el hogar de cerca de tres mil millones de consumidores e incluyen a algunas de las economías más dinámicas del mundo (Brilliant, 2010).

Para Bergsten (2007), quien apoya fuertemente la iniciativa FTTAP, el gran problema a resolver es probablemente la posición de China. Este autor considera que además de Estados Unidos y Japón, otros notables miembros de APEC están dispuestos a llevar a cabo negociaciones serias en este sentido, pero que existen otras preocupaciones, tales como: un posible efecto adverso sobre la Ronda de Doha y un retraso en la ejecución de las metas de Bogor propuestas por el mecanismo de APEC desde 1994.

Desde noviembre de 2011 se plantearon los lineamientos generales para transitar del TPP al FTTAP; éstos son: eliminar las barreras comerciales residuales a través de una programación definida, así como suprimir las normas e instituciones que inhiben el comercio transfronterizo, con el fin de ampliar los beneficios y de avanzar hacia una economía regional única. Según el discurso, no se pretende crear un bloque con miras a eliminar barreras comerciales inter- 
nas y conservarlas para quienes queden fuera del grupo, sino por el contrario, dar facilidad para que otros se adhieran de manera automática al tratado, a reserva de la aceptación y cumplimiento de sus términos (Drysdale, 2011).

Además de la asociación económica transpacífica, la nueva relación que Estados Unidos busca con Asia abarca otras áreas de las relaciones internacionales, como la estratégico-militar, tal como lo han manifestado sus dirigentes. Por otra parte, la apertura para aceptar la adhesión de futuros miembros y de acoplarse con otras iniciativas del Este de Asia tienen una clara intención de formar futuras alianzas (Dent, 2008: 134-138; Nye, 2013; New Zealand, Ministry of Foreign Relations and Trade, 2009; Drysdale, 2010; Palmer, 2011).

Desde antes de que Estados Unidos adquiriera un papel protagónico en el TPP, en los diferentes foros relacionados con ASEAN, China había mostrado su preocupación sobre la inclusión de Estados Unidos y Rusia en este mecanismo, quienes desde la perspectiva china podrían "debilitar el espíritu de cooperación del Este de Asia, debido a diferentes intereses estratégicos" (Zhang, 2012).

Al definirse los lineamientos y los temas a negociar en el TPP liderado ahora por Estados Unidos, China ha rechazado participar en las negociaciones. China considera que si bien Estados Unidos promueve el TPP como una especie de TLC de alto nivel para el siglo XXI, y a partir de éste pretende realizar un cambio fundamental en la naturaleza del mecanismo de APEC, también puede ser visto como una maniobra de Estados Unidos para debilitar la integración y la cooperación particular del Este de Asia, al diluir el papel central que ha tenido la ASEAN (y por consiguiente, China) en la construcción de una comunidad en el Este de Asia. Un indicio básico de ello es que varios miembros de ASEAN no están incluidos en el TPP (Zhang, 2012).

Para China, el acceso al TPP representa tanto oportunidades como desafíos. Como plantea Cai (2012), la expectativa de un mercado mucho más amplio que impulse las exportaciones y las importaciones de China es muy atractivo; sin embargo, para China es necesario clarificar el funcionamiento y las regulaciones de los servicios financieros. Además, no existe claridad sobre las intenciones geopolíticas de Washington. Tal parece que Estados Unidos está utilizando al TPP como parte de su estrategia en Asia y el Pacífico para contener a China.

Sin duda que para China la negativa de formar parte del TPP estriba en aceptar las reglas diseñadas por Estados Unidos sobre derechos de propiedad intelectual (IPR), normas laborales y de medio ambiente. Además, en términos comerciales China se vería obligada a reducir sus aranceles por abajo de 
9.8\% en un corto plazo, lo cual su economía aún no está en condiciones de soportarlo (Armstrong, 2011; Basu, 2013b).

La reticencia de China no carece de sentido. En noviembre de 2011 el presidente Obama planteó, por una parte, que Estados Unidos ve con beneplácito el ascenso de China, siempre y cuando se apegue a las reglas globales; $y$ por otra, manifestó su determinación de resistir el reto que representa China para el liderazgo de Estados Unidos en Asia. Manifestó que Estados Unidos, con el objeto de perpetuar un futuro para Asia "enmarcado en los valores y los intereses estadounidenses", resistirá a China con todos los elementos a su alcance, incluyendo la fuerza militar. El discurso del presidente Obama mostró que Estados Unidos ve a China como su principal rival estratégico, y que tal rivalidad va en un rápido aumento (Armstrong, 2011; White, 2011).

Por lo tanto, el principal desafío del TPP es la exclusión de China. Dado que China es el principal motor de la economía mundial, su exclusión del TPP plantea serias dudas sobre las posibilidades de éxito de este esquema de integración (Armstrong, 2011).

Para China, su incorporación al TPP es una decisión muy difícil, ya que es una de las principales economías del Regional Comprehensive Economic Partnership (RCEP). Por ahora, tanto el TPP como la RCEP se consideran vías paralelas de la integración económica regional en el Pacífico asiático. A largo plazo puede presentarse la posibilidad de que Estados Unidos y China encuentren un camino intermedio que integre los elementos del TPP y la RCEP. En el mejor de los casos esta opción podría lograrse en el contexto del FTAAP (Basu, 2013b).

\section{De los Tratados ASEAN+1 al Regional Comprehensive Economic Partnership (RCEP)}

Los avances del proyecto P4-TPP-FTAAP, impulsados por Estados Unidos desde finales de 2011, han puesto en peligro la preeminencia de China sobre el proceso de integración en el Este de Asia, el cual ha evolucionado partiendo de ASEAN como unidad central. China ha apostado desde hace más de 10 años a este esquema, para lo cual firmó un tratado de libre comercio con la región (ASEAN+1). Para China el TLC con ASEAN representa mucho más que un simple acuerdo comercial; constituye un marco general para la cooperación con los países de la ASEAN. Al poner en práctica este instrumento, China se ha convertido en el principal mercado para las exportaciones de los países de 
la región. Pero además, las áreas de cooperación han ido mucho más allá del comercio, para incluir el desarrollo de infraestructura, conectividad y capital humano (Zhang, 2010; Zhang, 2012).

A partir de este esquema China ha promovido la cooperación con Japón y Corea del Sur (ASEAN+3) y prácticamente ha aceptado trabajar en un proyecto de integración regional más amplio que incluya a los 10 países de la ASEAN, más Australia, China, India, Japón, Corea del Sur y Nueva Zelanda (ASEAN+6), todos los cuales ya han firmado por separado acuerdos de libre comercio con la Asociación, conocidos como ASEAN+1. Ahora se pretende convertir estos instrumentos de carácter bilateral en uno solo de carácter regional, conocido como Regional Comprehensive Economic Partnership (RCEP). ${ }^{9}$

La historia de RCEP se remonta a unos 10 años, cuando se propuso el estudio para establecer un tratado de libre comercio entre la ASEAN, China, Japón y Corea del Sur (conocido como ASEAN+3). Esto se complementó en 2007 con un análisis paralelo para lograr un tratado de libre comercio entre la ASEAN más China, Japón y Corea del Sur, además de Australia, India y Nueva Zelanda (ASEAN+6). Ambos procesos de estudio concluyeron en 2011, cuando la ASEAN propuso el concepto RCEP.

En la Cumbre del Este de Asia (East Asia Summit) de finales de 2012, los líderes de las economías de ASEAN+6 acordaron formalmente iniciar negociaciones del Tratado de Asociación Económica Integral Regional (RCEP), sobre bases menos rigurosas que otros instrumentos, en cuanto a tiempos y niveles de desgravación. Uno de los acuerdos fundamentales para la negociación de este nuevo mecanismo fue la adopción del regionalismo abierto, que en este caso plantea las opciones de poder aceptar nuevos miembros, así como de no impedir que miembros actuales puedan adherirse a otros grupos de libre comercio. De hecho, Australia, Brunei, Malasia, Nueva Zelanda, Singapur y Vietnam ya forman parte del grupo de países negociadores del TPP (Murray y Liam: 2012).

Con esta decisión salomónica, los integrantes del RCEP lograron destrabar un enredo institucional con un fuerte trasfondo histórico, y sobre todo político, relacionado con los liderazgos de dentro y fuera de la región. Varios analistas habían considerado que los rencores históricos, la lucha por hacer

9. Los participantes en las negociaciones del TLC RCEP tienen una población total de más de tres mil millones de personas y una cuota de comercio que se estima en alrededor de $27 \%$ del comercio global (sobre cifras de 2010 de la OMC), que cubre el PIB de alrededor de us $\$ 20$ billones de dólares (cifras de 2011 del FMI) (New Zealand Ministry of Foreign Affairs and Trade, 2013). 
prevalecer un determinado esquema y las tensiones territoriales de los últimos meses impedirían llegar a un acuerdo que lograra conciliar las posiciones de las economías involucradas, pero tal parece que ha predominado la cordura y el interés económico de sus integrantes. Hay que tener en cuenta que además del espíritu conciliador de los miembros de la ASEAN, los líderes de China, Japón y Corea del Sur buscaron tenazmente encontrar una fórmula de negociación. Los líderes del grupo han manifestado que el RCEP será instrumento moderno, de alto nivel de integración, de beneficio mutuo, de apertura y facilitación del comercio y las inversiones para contribuir al crecimiento económico y el desarrollo (New Zealand Ministry of Foreign Affairs and Trade, 2013; Rathus, 2009; Siew y Zainal, 2009).

Los puntos centrales de RCEP consisten en mantener el papel central de la ASEAN en la arquitectura económica regional, así como tratar de armonizar la "maraña" institucional de los distintos tratados de libre comercio firmados entre sus miembros. En principio, se pretende promover una mayor integración económica regional, eliminar progresivamente las barreras arancelarias y no arancelarias y garantizar la coherencia con las normas de la Organización Mundial del Comercio. De manera inmediata el RCEP se enfocará en los temas del comercio de bienes y servicios, de inversión, cooperación económica y técnica, propiedad intelectual, política de competencia y solución de controversias; más los temas adicionales que vayan surgiendo sobre la marcha.

De concretarse los objetivos planteados por RCEP, la región se convertiría en uno de los bloques comerciales más grandes del mundo, además de marcar una pauta en la batalla geopolítica para dar forma al futuro de las normas y estándares comerciales regionales.

Sin embargo, el RCEP tendrá que resolver otras cuestiones importantes si pretende estar a la altura de su potencial. Los detalles siguen siendo escasos, pero lo que sí sabemos de los Principios Rectores de RCEP es que se van a completar y a mejorar sustancialmente - no sustituir - los tratados ASEAN+1. Un elemento clave para medir la calidad del RCEP es la conocida (y temida) cláusula de "flexibilidad". La flexibilidad puede ser una bendición o maldición para la RCEP. Por una parte, podría ayudar a resolver los puntos muertos y proteger los intereses nacionales divergentes, pero por otra, también podría limitar el cambio o muchos avances necesarios (Menon, 2013).

Los miembros del RCEP, conscientes de que las grandes diferencias de desarrollo dentro de la ASEAN impiden la aplicación de políticas agresivas de liberalización comercial, consideran necesario otorgar una mayor flexibilidad 
y trato especial diferenciado para algunos de sus miembros. Y por otra parte, consideran necesaria la cooperación económica y tecnológica para reducir las diferencias de desarrollo. Este tipo de flexibilidad garantiza que ningún miembro tenga que adoptar políticas comerciales con las que no está de acuerdo, así como proteger de la competencia a las industrias sensibles. Esta concesión ha permitido integrar a los países menos desarrollados de la región y garantizar mayor número de miembros.

Se espera que las economías del Sudeste de Asia, orientadas a la exportación, se vean favorecidas con el acceso a los florecientes mercados nacionales de China, Japón y la India. De la misma manera, se espera que el RCEP contribuya a estimular la inversión de los países más desarrollados a los menos desarrollados y que se integren más plenamente en la actividad económica regional.

A diferencia del TPP, que exige una liberalización económica mucho más rápida y profunda de sus miembros, la RCEP incluye disposiciones para proteger los derechos laborales y normas ambientales, la reforma de las empresas estatales, la protección estricta de la propiedad intelectual, y eliminar agresivamente los aranceles (Murray y Liam, 2012).

Las negociaciones, que han tomado impulso durante 2013, se espera que concluyan a finales de 2015 y constituir así la Comunidad Económica de la ASEAN (AEC).

La velocidad en las negociaciones de la RCEP es crucial para mantener la centralidad de la ASEAN frente a otros procesos de integración regional (TLC China-Japón-Corea y el TPP). Además, existe la necesidad de consolidar los diferentes tratados de ASEAN +1 con el fin de armonizar las cuestiones relacionadas con aranceles, barreras no arancelarias, reglas de origen, facilitación comercial y la regulación del sector servicios; necesarias para continuar estimulando el crecimiento económico de la región (Basu, 2013b).

Para México es de primordial importancia seguir de cerca la evolución del RCEP, dado que China es su segundo socio comercial, sólo después de Estados Unidos, y que a diferencia de otros países latinoamericanos, mantiene un déficit comercial de grandes proporciones con la RPCh, así como balanzas negativas con el bloque de ASEAN, Japón y Corea. Hay que tener en cuenta, asimismo, que Chile, Perú y Costa Rica han firmado tratados de libre comercio con China; que Chile y Perú han hecho lo propio con Japón y que Colombia está en proceso de negociación de un Acuerdo de Asociación Económica con este país (MOFA, 2013). Esta circunstancia, aunada al hecho de la estrecha correlación productiva y comercial del Este de Asia, obliga a México a reconsiderar la 
conveniencia de negociar un tratado de libre comercio con la RPCh, tal como lo ha planteado el presidente Peña Nieto. Para el gobierno mexicano no será fácil abordar este tema, dadas las percepciones tan encontradas que existen sobre China en los sectores políticos, empresariales y sociales de México, las cuales se reflejan frecuentemente en los medios de comunicación (Cornejo, Haro Navejas y León-Manriques, 2013). Con Corea del Sur, desde hace varios años México ha venido negociando un TLC y sólo falta encontrar una fórmula de acercamiento que permita concluir las negociaciones. Aunque en el caso de este país, su posible incorporación al TPP haría innecesario para México negociar un tratado de carácter bilateral.

\section{El Pacífico latinoamericano}

Los países de América Latina, durante buena parte del siglo xx realizaron grandes esfuerzos de integración económica, con escasos o nulos resultados. Ahora, en el segundo decenio del siglo xxi surge la Alianza del Pacífico como una nueva propuesta para avanzar hacia estadios superiores de integración económica; aunque como en otros tiempos, se presenta el gran reto de resolver algunas contradicciones importantes con otros países de la región. América Latina tiene ante sí la tarea histórica de superar actitudes caudillistas y de "supremacía" nacional, que han impedido la coordinación de esfuerzos para lograr una auténtica y funcional integración económica regional.

La posición mexicana sobre esta cuestión es muy clara:

La estabilidad y prosperidad de esta región tiene beneficios o repercusiones directas para México [...] Hoy México tiene la oportunidad de desempeñar un papel activo para que los proyectos de integración y desarrollo panamericanos no queden en el tintero [...] Para México la integración comercial con América Latina no se limita a la profundización de los acuerdos o a la negociación de nuevos instrumentos comerciales, también incluye la convergencia de los tratados existentes (PND, 2013: 94).

\section{La Alianza del Pacífico}

A mediados de 2012 surgió un nuevo proyecto de integración económica en la ribera del Pacífico americano. Esta iniciativa de Colombia, Chile, México y Perú, que se ha dado a conocer como la Alianza del Pacífico (AP) surge a partir del 
grupo del Arco del Pacífico ${ }^{10}$ ante la falta de avances de éste. Por otra parte, las dificultades del Mercosur, originadas principalmente por la aplicación de prácticas proteccionistas unilaterales, al margen de las negociaciones comerciales, de algunos de sus miembros (aun en contra de sus socios), motivó a Colombia, Chile, México y Perú a buscar un nuevo esquema de integración regional.

Los cuatro países que impulsan la AP comparten los principios y aceptan las normas de los regímenes internacionales financiero y comercial; son afines en cuanto a sus políticas económicas, las cuales se reflejan en el alto nivel de apertura comercial; coinciden en cuanto a sus condiciones socioeconómicas y culturales y en el interés manifiesto de sus dirigentes de convertirla en una plataforma de articulación económica (productiva y comercial) y de proyección al mundo, con especial énfasis en el Asia Pacífico. De ahí los objetivos de la Declaración de Lima, que en esencia planeó lograr en un corto plazo la libre circulación de bienes, inversiones, servicios y personas; así como crear un sistema universitario regional y una bolsa de valores conjunta (Secretaría de Economía, 2012).

La Alianza del Pacífico, que hasta hoy la integran los cuatro países signatarios de la iniciativa, es un mercado en crecimiento con una población conjunta de 209 millones de habitantes. El PIB de sus miembros alcanza los 2.01 mil millones de dólares y comprende $36 \%$ respecto del PIB de la región de América Latina y el Caribe, con lo cual representa la octava economía a nivel mundial. Con $50 \%$ del comercio total de América Latina, es la séptima potencia exportadora a nivel mundial (emol.economia, 2013; KINC News, 2013). ${ }^{11}$

Después de dos años de negociaciones, en la reunión de ministros realizada el 25 de agosto de 2013 en Playa del Carmen, México, se lograron los acuerdos previstos sobre libertad de circulación de bienes, servicios, capitales y personas. En particular se acordó desgravar, en forma inmediata, tras la firma del tratado, $92 \%$ de las partidas arancelarias; el restante $8 \%$ se proyecta desgravar a corto y mediano plazos (Méxicoxport, 2013).

El secretario de Economía de México comentó que el acuerdo económicocomercial alcanzado ha dejado relegados "muy pocos productos de un alto

10. El Arco del Pacífico, creado en 2007 e integrado por Chile, Colombia, Costa Rica, Ecuador, El Salvador, Guatemala, Honduras, México, Nicaragua, Panamá y Perú, tenía como finalidad coordinar una estrategia continental para la relación comercial con las economías más sólidas y también las emergentes de Asia Pacífico. Sin embargo, como en otros casos, ante la falta de avances concretos se convirtió en un membrete (Muñoz y López, 2013).

11. Para más detalles sobre la situación socioeconómica de la AP, véase el Anexo 1. 
nivel de sensibilidad" y precisó que estos artículos representan "menos de $1.4 \%$ del comercio total”, los cuales serán ajustados a diferentes plazos (KINC News, 2013).

Durante la rueda de prensa en la cual se anunció la conclusión de las negociaciones, se informó que el próximo 25 de septiembre los presidentes de los cuatro países promoverán la Alianza del Pacífico en el marco de la Asamblea General de las Naciones Unidas, aprovechando la asistencia de los presidentes de los países signatarios e importantes líderes empresariales del mundo.

Además, con el fin de agilizar el proceso de integración, las legislaturas de los países de la AP han constituido la Comisión Interparlamentaria de la Alianza del Pacífico; con ello los legisladores están preparando las condiciones para discutir y aprobar, de manera más expedita, los acuerdos que logren los poderes ejecutivos de los cuatro países.

La VII Cumbre de la Alianza del Pacífico, realizada en Santiago de Cali, Colombia, del 20 al 24 de mayo de 2013, representó un avance significativo en la consolidación de esta iniciativa. A la Cumbre asistieron los presidentes de los países miembros: México, Chile, Perú y Colombia. Por parte de los países observadores asistieron el primer ministro de Canadá, ${ }^{12}$ el presidente del Gobierno de España, la presidenta de Costa Rica, el presidente de Panamá ${ }^{13}$ y el presidente de Guatemala, así como delegaciones ministeriales de Uruguay, Australia, Japón, Portugal, Nueva Zelanda y República Dominicana; todos con el estatus de miembros observadores.

Entre los acuerdos más importantes, destacan la creación de una visa Alianza del Pacífico para promover el turismo de ciudadanos de países terceros dentro de la región y los lineamientos para la inclusión de nuevos países miembros, comenzando con Costa Rica. Asimismo, se formalizó la inclusión de 16 naciones en calidad de observadoras. El grupo quedó formado por España, Panamá, Australia, Nueva Zelanda, Uruguay, Canadá, Japón y Guatemala; más los nuevos de Ecuador, El Salvador, Francia, Honduras, Paraguay, Portugal y República Dominicana (El País, 2013). Y en la pasada reunión de la AP, celebrada en Villa de Leyva, Colombia, la canciller de este país dio cuenta de

12. Canadá ha estado interesada en la Alianza, desde sus orígenes, puesto que ya cuenta con tratados de libre comercio con todos los países miembros, al igual que con Costa Rica.

13. Dado que uno de los requisitos para formar parte de la alianza es tener acuerdos comerciales con todos sus miembros, Panamá gestiona por separado, tanto con México como con Colombia, tratados de libre comercio. El primero de mayo de 2012 entró en vigor un convenio firmado con Perú, mientras que con Chile ya tiene acuerdos. 
la petición de Estados Unidos, China, Corea del Sur y Turquía de participar en calidad de observadores. Lo anterior da cuenta del interés manifiesto de actores importantes del sistema internacional, de participar y conocer de cerca la evolución de este esquema de integración.

Por su parte, el sector privado de los cuatro países ha manifestado su interés en el esquema de la AP. En la cumbre de Cali, de mayo de 2013, los representantes del sector privado de la región realizaron reuniones específicas, de manera paralela a los encuentros de los funcionarios gubernamentales. En este foro, el Consejo Empresarial de la Alianza del Pacífico entregó una serie de propuestas a los presidentes, entre las que se destacan la necesidad de adoptar medidas de facilitación comercial de última generación; de implementar certificados de origen de forma digital, de realizar la interconexión regional de las ventanillas únicas de comercio exterior y autorizar la acreditación de operadores económicos (BID, 2013).

Un mes después (el 19 y 20 de junio), tal como se había previsto en la Cumbre de Cali, tuvo lugar la primera gran macrorrueda de negocios de la Alianza del Pacífico, la cual promovió principalmente los encadenamientos productivos para llegar a los países asiáticos. La reunión empresarial dejó un balance de us $\$ 90$ millones en negocios concretados en casi 4,000 citas entre unos 700 empresarios de los cuatro países. El sector que generó las mayores expectativas fue el de productos alimenticios (53\%), seguido por manufacturas (25\%), prendas de vestir (13.8\%) y servicios (7.1\%). En total acudieron a la macrorrueda 215 empresas compradoras: 77 de Colombia, 54 de México, 44 de Chile y 40 de Perú, las cuales se encontraron con la oferta de 374 empresas exportadoras representadas por 467 empresarios de los cuatro países: 137 de Colombia, 96 de Chile, 80 de México y 61 de Perú (El Mundo, 2013; Morales, 2013).

Estados Unidos, en voz de la secretaria adjunta para Asuntos del Hemisferio Occidental, Roberta S. Jacobson, ha manifestado su apoyo a esta iniciativa de integración. Específicamente afirmó que "según analistas y especialistas en la materia, la Alianza del Pacífico es el bloque comercial líder en exportaciones y comercio exterior de toda América Latina y avanza a convertirse en el bloque comercial más grande y ambicioso de Latinoamérica" (Américaeconomía, 2013a). Desde Canadá la opinión es que la Alianza del Pacífico ha evolucionado rápidamente para convertirse en una alternativa a otros bloques comerciales de la región, los cuales se han entrampado en luchas internas y nuevas medidas proteccionistas (Crowe y Kozak, 2013). 
Con la conformación de la Alianza del Pacífico, en América Latina se van configurando dos proyectos de integración regional, los cuales representan dos maneras de entender y practicar las relaciones económicas. Por un lado están el Mercosur, ${ }^{14}$ la Alianza Bolivariana de las Américas (ALBA), la Unión de Naciones Sudamericanas (Unasur) y la Comunidad de Estados Latinoamericanos y del Caribe (CELAC), las cuales se centran más en objetivos políticos que de integración económica. En el otro espectro está la propuesta de la Alianza del Pacífico, que pone gran énfasis en las medidas de liberalización comercial. De acuerdo con la Declaración de Lima la AP se propone "alentar la integración regional, así como un mayor crecimiento, desarrollo y competitividad" de las economías de sus países, así como incorporar al proceso a los países de la región que "compartan la voluntad de alcanzar las metas de esta Alianza”. Sin embargo, el componente político no está exento, puesto que también establece como requisito esencial la vigencia del Estado de derecho, de la democracia y el orden constitucional.

Más allá del componente político-ideológico de la AP y los otros esquemas de integración latinoamericanos, existen también diversas contradicciones de carácter funcional que involucran a la mayoría de los países de la región. Los cuatro miembros de la Alianza del Pacífico han firmado tratados de libre comercio con Estados Unidos y Canadá, ${ }^{15}$ por un lado, y acuerdos preferenciales con el Mercosur, por otro. Chile tiene una economía íntimamente conectada con la de Argentina, mientras que Colombia exporta gran parte de su producción industrial a Venezuela. Por su parte, Perú, Chile y Colombia son parte de Unasur y, con sus diferencias, han estado alineados en la integración política promovida desde esta organización. Además, desde la muerte de Néstor Kir-

14. Después de 22 años, el cumplimiento de los objetivos del Mercosur en buena medida siguen pendientes y en algunos aspectos las políticas económicas nacionalistas de sus miembros los alejan de sus compromisos. Los objetivos del Mercosur, planteados desde su origen en el Tratado de Asunción de 1991, son: establecer la libre circulación de bienes, servicios y factores productivos entre los países miembros mediante, entre otras medidas, la eliminación de los derechos aduaneros y restricciones paraarancelarias; fijar una tarifa externa común, adoptar una política comercial común en relación con terceros países o bloques regionales, y coordinar las posiciones de sus miembros en foros comerciales de la región e internacionales; coordinar las políticas macroeconómicas y de comercio exterior, agrícolas, industriales, fiscales, monetarias, cambiarias y de capitales, de servicios, aduanera, de transportes y comunicaciones, y otras que se acuerden en el futuro, con el fin de asegurar condiciones adecuadas de competencia entre los Estados parte (El País, 2011).

15. Además, Canadá tiene tratados comerciales con Panamá y Costa Rica, dos países observadores que apuntan a convertirse en miembros de la AP. 
chner, la Secretaría General de Unasur quedó en manos de Colombia. México y Chile son miembros de la OCDE.

Por otra parte, México, junto con el resto de los países de América Latina, forma parte de CELAC, considerada por sus miembros como un espacio de unidad en oposición a la presencia de Estados Unidos en la región. En la última reunión de la CELAC la presidencia pro-témpore pasó de Chile a Cuba. Lo anterior pone de manifiesto las contradicciones que tendrán que resolver los países de América Latina para hacer viable un proyecto que puede aprovechar las ventajas de las relaciones históricas de sus países o avanzar contra marea, frente a la oposición de los países que rechazan, como principio y en la práctica, las políticas de liberalización comercial propuestas por organismo internacionales como la OMC, así como el mayor involucramiento de Estados Unidos en la definición de las políticas comerciales de la región.

Recientemente (junio de 2013) el presidente Piñeira de Chile puntualizó algunos aspectos relacionadas con los dos proyectos de integración en América Latina. La Alianza del Pacífico - señaló- "no es una cosa ideológica, no es una respuesta al ALBA, que apunta menos a la integración económica y más a la coordinación política [...], pero obviamente que es una comunidad con una visión política distinta" a la que tienen los países del ALBA. Respecto del Mercosur, afirmó: "Nosotros buscamos una integración regional que no sea una alternativa a la integración en el mundo, sino al revés, una manera de acelerarla [...] Desde ese punto de vista, la visión de la Alianza del Pacífico y la visión del Mercosur son definitivamente distintas". Y agregó que los socios de la Alianza esperan lograr muy pronto la total liberalización del intercambio de bienes en común, y que los ciudadanos de los países miembros puedan transitar libremente (sin visas) por la región, y que incluso en un futuro cercano puedan trabajar libremente en cualquiera de los cuatro países (América-economía, 2013b). Otra opinión muy optimista es la del ministro peruano de Comercio Exterior y Turismo, José Luis Silva, quien afirma que "el objetivo es tener una zona de libre comercio, similar a la de la Unión Europea, en donde las personas, los capitales y las mercancías se puedan mover libremente" (El País, 2013).

No obstante el entusiasmo sobre los proyectos de integración de la AP, existe cierto grado de incertidumbre sobre sus resultados concretos: el principal es el amplio historial de proyectos fracasados en América Latina. Una perspectiva más optimista se fundamenta en la amplia red de tratados 
y acuerdos comerciales y de inversión ya existentes de carácter bilateral y regional, los cuales requieren de grandes esfuerzos de armonización.

Para México la Alianza del Pacífico representa una asociación estratégica, puesto que, además de compartir con Colombia, Chile y Perú la perspectiva sobre el modelo de integración económica, el nivel de intercambio comercial entre los cuatro países es muy significativo dentro del contexto latinoamericano. Un tercio de las exportaciones mexicanas hacia América Latina tienen como destino los países de la AP y $23 \%$ de las compras mexicanas de América Latina provienen de esta región. Los tratados previos de libre comercio firmados por los cuatro países han propiciado el aumento del intercambio entre éstos (Secretaría de Economía, 2012). Ahora, con la implementación de un tratado regional se esperan incrementos adicionales en la relación comercial y productiva dentro de la región, y de ésta con el resto de los países; en especial con Asia. La coincidencia de los cuatro países en cuanto a la promoción del libre comercio, el impulso a la competitividad, el desarrollo de las cadenas de valor, los encadenamientos productivos de las empresas y la generación de empleo, auguran el establecimiento de una relación más fructífera para los miembros de la AP.

\section{Conclusiones}

En la negociación de los procesos de integración económica en el Pacífico asiático y el Pacífico latinoamericano, como parte de la dinámica del sistema internacional, prevalece la perspectiva e intereses de los grandes actores, en especial de Estados Unidos y

En la negociación de los procesos de integración económica en el Pacífico asiático y el Pacífico latinoamericano, como parte de la dinámica del sistema internacional, prevalece la perspectiva e intereses de los grandes actores, en especial Estados Unidos y China" China. En esta correlación de pesos y contrapesos, el resto de actores internacionales se ven obligados a tomar partido intentando, por lo general, evitar confrontaciones con los poderes hegemónicos de alcance regional o global. En cada uno de los actores internacionales, la identidad, el interés nacional y la perspectiva de los tomadores de decisiones son clave para conocer e interpretar el tipo, nivel y momento de su participación en los procesos. 
Las nuevas tendencias de integración entre los países del Pacífico asiático (más Australia y Nueva Zelanda) y los del Pacífico latinoamericano, representan esquemas de cooperación intermedia entre los instrumentos de carácter multilateral promovidos por la OMC y la proliferación desmedida de tratados bilaterales de comercio e inversión (spaghetti bowl).

De los preceptos centrales de los regímenes internacionales comercial y financiero se derivan los temas en negociación dentro de los mecanismos del TPP, el RCEP y la AP, entre los que se cuentan: reglas de origen, barreras técnicas al comercio, inversión extranjera, cuidado del medio ambiente, pequeñas y medianas empresas, competitividad y cadenas de abastecimiento, entre otros. En los casos del TPP y del RCEP, sobresalen por su dificultad los derechos de propiedad intelectual, transparencia tecnológica, coherencia regulatoria, empresas estatales, políticas de competitividad, relaciones laborales, medio ambiente, y en especial los derechos de las industrias farmacéutica y electrónica. Todos estos temas, algunos de los cuales se mencionan de manera general en este trabajo, requerirán de investigaciones más profundas, una vez que se conozcan con detalle las modalidades de los acuerdos alcanzados y se analicen las implicaciones para los países miembros y sus relaciones con otras economías y con otros mecanismos de integración, como el Mercosur, el ALBA, el TLCAN y la Unión Europea.

Como se ha señalado en el cuerpo del trabajo, los actores que participan en los esquemas de integración del TPP, RCEP y AP, no proponen un cambio al paradigma tradicional del sistema económico, sino sólo ajustes al mismo para operarlo a su favor, considerando la obtención de un saldo positivo en la correlación costo-beneficio. Esto es claro aún para China, que desde su ingreso a la OMC en 2001, se ha visto especialmente beneficiada de las reglas del juego y de la aceptación del statu quo internacional. Los actores que no están directamente involucrados en las negociaciones participan como observadores activos o pasivos de los procesos, dadas sus estrechas relaciones económicas y políticas con los miembros del TPP, RCEP y AP.

En el caso de México, cabe resaltar, además, la coincidencia de las administraciones de Calderón y de Peña Nieto respecto de la estrategia de negociación dentro de la Alianza del Pacífico y el TPP. Dichos proyectos se enmarcan en la estrategia de las negociaciones comerciales internacionales del país que, trasciende los límites de los periodos sexenales. Es de esperar que los cambios políticos en los demás países de la AP no cambien la postura respecto de los acuerdos negociados. 
Hay que tener en cuenta que los tres procesos de integración analizados comparten la característica de ser negociaciones cupulares sin participación visible, ni información suficiente hacia la sociedad civil; una reminiscencia de la diplomacia tradicional. En términos discursivos, los documentos y las declaraciones de los negociadores se presentan como nuevas plataformas de trabajo en la búsqueda de relaciones más equitativas y mutuamente beneficiosas. En la práctica, los intereses de los países participantes dificultan el logro de los consensos. Aunque al mismo tiempo, cada vez existe una mayor interrelación comercial y productiva entre ellos. Los flujos globales de comercio e inversión y la participación formal de muchos países en esquemas paralelos de integración regional, son condiciones objetivas sobre las cuales es indispensable trabajar.

La urgencia de alcanzar los acuerdos básicos y firmar los documentos marco es muy evidente. En la práctica, las dificultades de alcanzar un tratado integral y de alto estándar, son mayores entre los 12 miembros del TPP, que entre los cuatro países de la AP. Los primeros se enfrentan a una gran diversidad en términos de tamaño, población y desarrollo económico, mientras que entre los países de la AP las diferencias en el grado de apertura de sus economías (medido en términos de nivel arancelario), PIB per cápita y densidad de población no son tan amplios. La diversidad de los miembros del RCEP la están mitigando con la temida cláusula de flexibilización, que podría limitar el alcance del propio instrumento.

Por su parte, los países miembros de la Alianza del Pacífico han manifestado su aceptación de ampliar su membresía con la inclusión de Costa Rica y Panamá y de contribuir al proceso global de integración económica y comercial, con especial énfasis en la región Asia-Pacífico. Ello permite interpretar que la AP, como lo fue en su momento el P-4, pueda convertirse en un esquema transitorio para la negociación de una plataforma macrorregional mucho más amplia, como la propuesta por Estados Unidos del Tratado de Libre Comercio de APEC (FTAAP).

\section{Referencias bibliográficas}

América-economía (2013a), "Estados Unidos destaca a la Alianza del Pacífico como modelo pragmático y exitoso", América-economía, 25 de abril, disponible en: http://www.americaeconomia.com/economia-mercados/ 
finanzas/eeuu-destaca-la-alianza-del-pacifico-como-modelo-pragmaticoy-exitoso

_- (2013b), "La Alianza del Pacífico acelera la integración política y económica de sus cuatro socios", América-economía, 9 de junio, disponible en: http://www.americaeconomia.com/economia-mercados/comercio/ la-alianza-del-pacifico-acelera-la-integracion-politica-y-economica-de-su Armstrong, Shiro (2011), "China's participation in the Trans-Pacific Partnership", East Asia Forum, 11 de diciembre, disponible en: http://www. eastasiaforum.org/2011/12/11/china-participation-in-the-trans-pacificpartnership

Basu, Das Sanchita (2013a), "Moving ASEAN+1 FTAs towards an effective RCEP”, ISEAS Perspective, núm. 29, 10 de mayo.

- - (2013b), "The Trans-Pacific Partnership as a tool to contain China: Myth or reality?", ISEAS Perspective, Singapur, disponible en: http://www.iseas. edu.sg/documents/publication/iseas_perspective_2013_31-the-tpp-asa-tool-to-contain-china-myth-or-reality.pdf

Bergsten, C. Fred (2007), "China and Economic Integration in East Asia: Implications for the United States", Policy Briefs in International Economics, núm. PB07-3, marzo. Recuperado de: http://www.iie.com/publ.ications/ pb/pb07-3.pdf

BID (2013), "Presidentes de la Alianza del Pacífico acuerdan desgravación total de aranceles", BID, disponible en: http://www.iadb.org/es/noticias/ comunicados-de-prensa/2013-05-24/alianza-del-pacifico,10463.html

Brewer, W. (2010), MFAT P4 Trans-Pacific Partnership. MFAT P4 Trans-Pacific Partnership, disponible en: http://www.mfat.govt.nz/trade-and-economic-relations/trade agreements

Brilliant, Myron (2010), "A Free Trade Area of the Asia-Pacific: An Idea with Merit, but is it Feasible?", The Brookings Institution, disponible en: http:// www.brookings.edu/opinions/2007/09northeastasia_brilliant.aspx

Cai, Penghong (2012), The Trans-Pacific Partnership: A Chinese Perspective, disponible en: http://www.pecc.org/resources/doc_view/1752-the-transpacific-partnership-a-chinese-perspective-ppt

Cornejo, Romer, Francisco Javier Haro Navejas, y José Luis León-Manríquez (2013), "Trade Issues and Beyond: Mexican Perceptions on Contemporary China", Latin American Policy, 4(1), pp. 57-75.

Crowe, Darcy, y Robert Kozak (2013), "Canadá mira a Alianza del Pacífico para impulsar el comercio", The Wall Street Journal, 23 de mayo, disponible en: 
http://online.wsj.com/article/sb1000142412788732347530457850128 1242623620. html

Cuevas, Rodrigo (2013), Negociaciones del Acuerdo Trans-Pacific-Partnership, Chile: Universidad de Chile-Instituto de Estudios Internacionales, disponible en: http://www.iei.uchile.cl/noticias/93233/negociaciones-delacuerdo-trans-pacific-partnership

Dent, M. Christopher (2008), East Asian Regionalism, Nueva York: Routledge. Drysdale, Peter (2010), "Is the Trans-Pacific Partnership Idea a Dead End?", East Asia Forum, 15 de noviembre, disponible en: http://www.eastasiaforum.org/2010/11/15/is-the-trans-pacific-partnership-idea-a-deadend/\#more-15232

- - (2011a), "Are here real dangers in the Trans-Pacific Partnership idea?", East Asia Forum, 18 de abril, disponible en: http://www.eastasiaforum. org/2011/04/18/are-there-real-dangers-in-the-trans-pacific-partnershipidea

(2011b), “Obama's regional summitry", East Asia Forum, 14 de noviembre, disponible en: http://www.eastasiaforum.org/2011/11/14/obamasregional-summitry

——, y Kenichi Ishigaki (2007), "New Issues in East Asian Economic Integration", en Drysdale, Peter, y Kenichi Ishigaki, Asia-Pacific Economic Cooperation. Critical Perspectives on the World Economy, Nueva York: Routledge, pp. 411-420.

El Mundo (2013), "Noventa millones de dólares en negocios ha dejado la Alianza del Pacífico", El Mundo, 21 de junio, disponible en: http://www. elmundo.com/portal/noticias/economia/ 90_millones_de_dolares_en_ negocios_ha_dejado_la_alianza_del_pacifico.php

El País (2013), "La Alianza del Pacífico y la cumbre de Cali", El País, 10 de junio, disponible en: http://economia.elpais.com/economia/2013/06/10/ actualidad/1370850899_293631.html

emol.Economía (2013), "Chile, Colombia, México y Perú alcanzan acuerdo para liberar el 100\% de su comercio", disponible en: http://www.emol. com/noticias/economia/2013/08/27/616631/ chile-colombia-mexico-yperu-alcanzan-acuerdo-para-liberar-el-100-de-su-comercio.html

Feldman, Elliot J. (2010), "China's Status As A Non-Market Economy”, ChinaUS Trade Low, viewed, 6 de marzo, disponible en: http://www.chinaustradelawblog.com/ 
Fergusson, F. Ian (coord.) (2013), The Trans-Pacific Partnership Negotiations and Issues for Congress, Washington, Congressional Research Service, disponible en: www.crs.gov

Gallagher, K. (2008), “Competing for America's Business", The Guardian, 3 de septiembre, disponible en: http://www.guardian.co.uk/commentisfree/2008/sep/03/useconomy.mexico

(2011), "China Discovers Latin America", Berkeley Review of Latin American Studies, otoño, disponible en: http://www.ase.tufts.edu/gdae/pubs/ rp/gallagherbrlas fall2011.pdf

Gobierno de la República, México (2013), Plan Nacional de Desarrollo 2013-2018. V. México con responsabilidad global, México: Gobierno de la República.

Hernández Hernández, Roberto (2012), "Economic Liberalization and Trade Relations between Mexico and China", Journal of Current Chinese Affairs, 41(1), pp. 49-96, disponible en: www.giga-journal-family-org

IMF (2011), "World Economic Outlook", International Monetary Fund, disponible en: http://www.imf.org/external/pubs/ft/weo/2011/02/weodata/ weorept.aspx? sy $=2011 \&$ ey $=2016 \&$ scsm $=1 \&$ ssd $=1 \&$ sort $=$ country\&d $s=. \& b r=1 \& c=924 \& s=$ ngdpd $\% 2 c n g d p d p c \% 2 c p p p g d p \% 2 c p p p p c \% 2 c b$ ca_ngdpd\&grp $=0 \& a=\& p r . x=83 \& p r . y=18$

KINC News (2013), "La Alianza del Pacífico logra 'hito importante' al cerrar acuerdo económico", disponible en: http://www.tvkinc.com/2013/08/27/ la-alianza-del-pacifico-logra-hito-importante-al-cerrar-acuerdo-economico/

Latin American Herald Tribune (2011), "Mexico Poised to Restrict Import of Chinese Footwear", Latin American Herald Tribune, 8 de septiembre, disponible en: http://www.laht.com/article.asp?ArticleId=423566\&Cat egoryld $=14091$

Menon, Jayant (2013), "The challenge facing Asia's Regional Comprehensive Economic Partnership", East Asia Forum, 23 de junio, disponible en: http://www.eastasiaforum.org/2013/06/23/the-challenge-facing-asiasregional-comprehensive-economic-partnership

Méxicoxport (2013), "Promoverán Alianza del Pacífico en Nueva York", Méxicoxport, 29 de agosto, disponible en: http://www.mexicoxport.com/ noticias/7769/promoveran-alianza-del-pacifico-nueva-york

Mfa (2010), China and México. Bilateral Relations, Ministry of Foreign Affairs of the People's Republic of China, 26 de agosto, disponible en: http:// www.mfa.gov.cn/eng/wjb/zzjg/ ldmzs/gjlb/3508/t17382.htm

Middlebrook, J. Kevin, y E. Zepeda (2003), "On the Political Economy of Mexican Development Policy”, en Middlebrook, J. Kevin, y E. Zepeda 
(eds.), Confronting Development: Assessing Mexico's economic and social policy challenges, Stanford: Stanford University Press.

Ministerio de Relaciones Exteriores de Chile (2006), Acuerdo Estratégico Transpacífico de Asociación Económica, Chile: Dirección General de Relaciones Económica Internacionales.

Mofa (2013), Free Trae Agreement (FTA) and Economic Partnership Agreement (EPA), Japón: Ministry of Foreign Affairs of Japan, disponible en: http:// www.mofa.go.jp/policy/economy/fta/index.html

Morales, Roberto (2013), "Sacan jugo a la Alianza del Pacífico", El Economista, 20 de junio, disponible en: http://eleconomista.com.mx/industrias/2013/06/20/sacan-jugo-alianza-pacifico

Moran, T. (2011), "Foreign direct investment in China: Trading competitiveness for access?", East Asia Forum, 11 de abril, disponible en: http://www. eastasiaforum.org/ 2011/04/11/foreign-direct-investment-in-chinatrading-competitiveness-for-access

Murray, Hieber, y Liam Hanlon (2012), "ASEAN and Partners Launch Regional Comprehensive Economic Partnership", The Center for Strategic and International Studies, 7 de diciembre, disponible en: http ://csis.org/publicación/asean-y-parejas-launch-regional-global-económico-asociación

New Zealand Ministry of Foreign Affairs \& Trade (2009), Trans-Pacific Strategic Economic Partnership Agreement, disponible en http://www.mfat.govt.nz/ Trade-and-Economic-Relations/Trade-Agreement

- - (2013), Regional Comprehensive Economic Partnership (RCEP), disponible en: http://www.mfat.govt.nz/Trade-and-Economic-Relations/2-TradeRelationships-and-Agreements/rcep/

Nye, S. Joseph (2004), Soft Power. The Means to Success in World Politics, Nueva York: Public Affairs.

- - (2013), "Joseph Nye Commentary: American Power in 21st Century", Korea Times, disponible en: http://www.hks.harvard.edu/news-events/ news/news-archive/american-power-21st-century

Palmer, Doug (2011), "U. S. partner set target for Trans-Pacific trade deal", Reuters, 20 de mayo, disponible en: http://www.reuters.com/article/2011/05/20/us-usa-apec-idustre74h7mv20110520

Rathus, Joel (2009), "East Asia Community: Little chance of a breakthrough at the Trilateral Summit", East Asia Forum. Recuperado de: http://esatasiaforum.org 
Rosenzweig Mendialdúa, Francisco L. (2012), "México y su ingreso al Acuerdo de Asociación Transpacífico", Revista de Derecho Económico Internacional, 3(1), pp. 89-96.

Sally, Razeen (2013), "Global value chains, trade policy and Asia", East Asia Forum, 13 de junio, disponible en: http://www.eastasiaforum. org/2013/06/13/global-value-chains-trade-policy-and-asia/

Secretaría de Economía (2012), Alianza del Pacífico. Memorias documentales, México: Secretaría de Economía/Administración Pública Federal 2006-2012.

Siew Yean Tham and Zainal Abidin Mahani, (2009) ASEAN-Plus Six as a Vehicle for Regional Integration, from Institute of Malaysian and International Studies (IKMAS), University Kabangsaan, Malaysia. Disponible en: http:// www.isis.org.my/files/events/MZA\&TSY_Taipei_280907.pdf.

University Kabangsaan (s/f). Regional Integration, Malasia: University Kabangsaan-Institute of Malaysian and International Studies (IKMAS), disponible en: http://www.isis.org.my/files/ events/mza\&tsy_taipei_280907.pdf

Universopyme (2008), "Firman acuerdo cuotas compensatorias México y China", Universopyme, disponible en: http://www.universopyme.com. $\mathrm{mx} /$ index.php?option=com_content\&task=view\&id=2229\&Itemid=136 Ustr (2013), Trans-Pacific Partnership Negotiations Maintain Strong Momentum, Estados Unidos: Office of the United States Trade RepresentativeExecutive Office of the President, 24 de mayo, disponible en: http:// www.ustr.gov/about-us/press-office/press-releases/2013/may/tppnegotiations-strong-momentum

White, Hugh (2011), "America and China: Strategic choices in the Asia Century", East Asia Forum, 19 de febrero, disponible en: http://www. eastasiaforum.org/2012/02/19/america-and-china-strategic-choicesin-the-asian-century/

Williams, R. Brock (2013), Trans-Pacific Partnership (TPP) Countries: Comparative Trade and Economic Analysis, Washington: Congressional Research Service, disponible en: www.crs.gov

Zhang, Yunling (2010), China and the Asian Regionalism, Singapur: World Scientific Publishing, Co.

-_ (2012), “China's regional and global power”, East Asia Forum, 6 de febrero, disponible en: http://www.eastasiaforum.org/index.php?s=zhng+yunling 


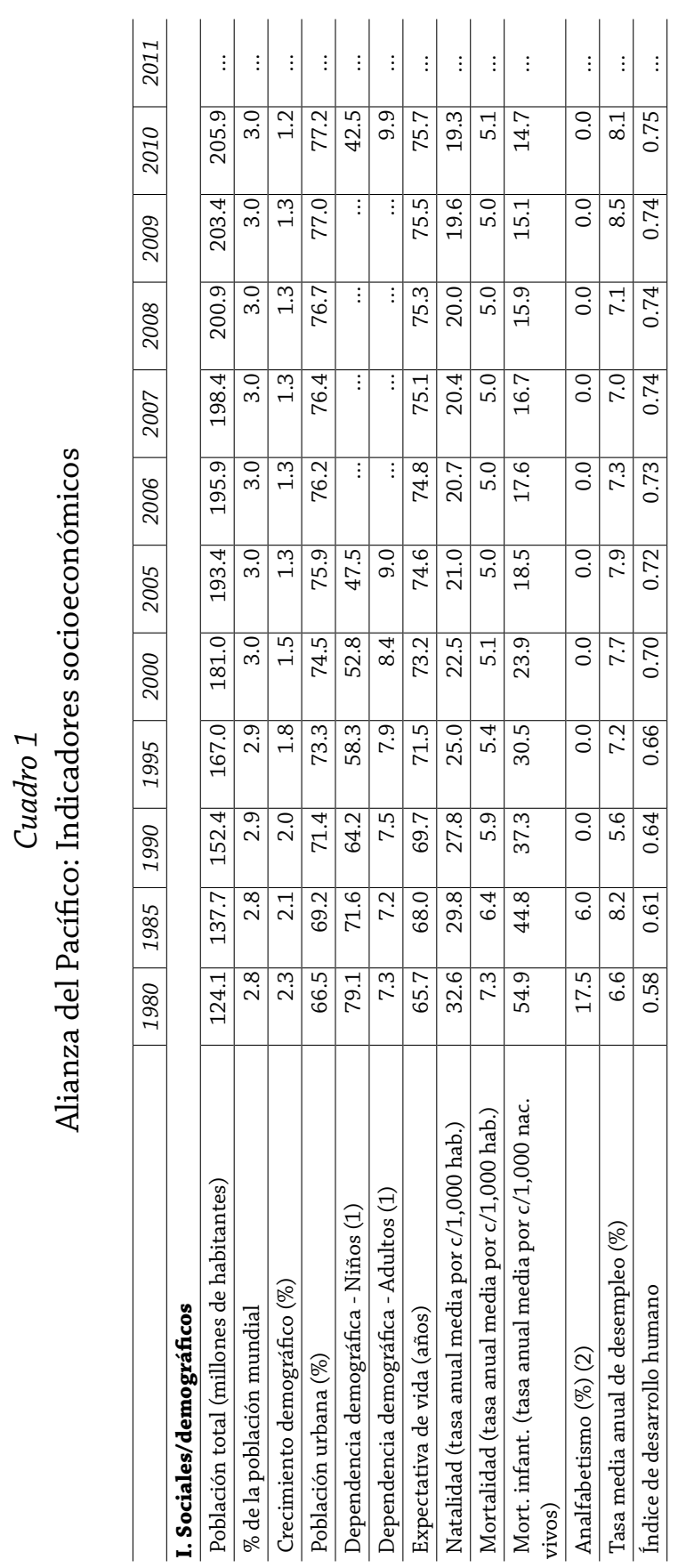




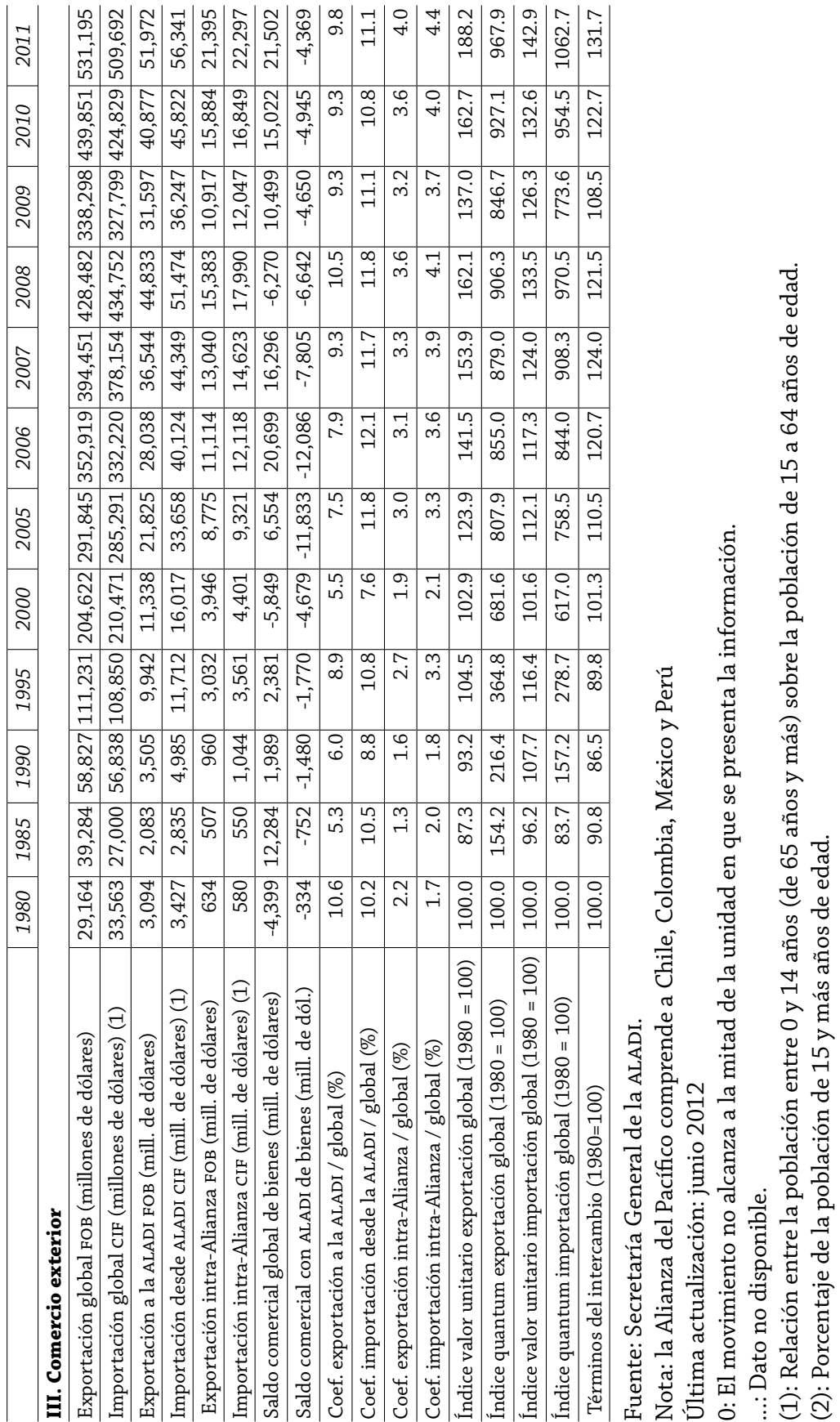




\section{EDITED BY \\ Beatriz Carrillo}

and

David S.G. Goodman

\section{China's Peasants and Workers: Changing Class Identities}

